\title{
Effect of Anisotropic Thermal Conductivity of Graphite Flakes and Interfacial Thermal Resistance on the Effective Thermal Conductivity of Graphite Flakes/ Aluminum Composites
}

\author{
Yan Zhao ${ }^{1, * 1}$, Kenjiro Sugio ${ }^{1, * 2}$, Yongbum Choi ${ }^{1}$, Sasaki Gen ${ }^{1}$, Zhefeng $\mathrm{Xu}^{2}$ and Jinku $\mathrm{Yu}^{2}$ \\ ${ }^{1}$ Graduate School of Engineering, Hiroshima University, Higashi-Hiroshima 739-8527, Japan \\ ${ }^{2}$ State Key Laboratory of Metastable Materials Science and Technology, Yanshan University, Qinhuangdao 066004, Hebei, P. R. China
}

\begin{abstract}
The effective thermal conductivity (ETC) of graphite flake (GF)/Al composites is significantly influenced by the anisotropic thermal conductivity of GFs and the interfacial thermal resistance between both components. A two-dimensional (2D) image-based simulation was used in this study to investigate the effect of both the orientation of GFs and interfacial thermal resistance on the ETC of the composite. 10 vol\% $\mathrm{GF} / \mathrm{Al}$ and $20 \mathrm{vol} \% \mathrm{GF} / \mathrm{Al}$ composites were fabricated via spark plasma sintering. The microstructure ETC, and the relative density of the GF/Al composites were determined. The experimental ETCs were smaller than calculated ETCs using the rule of mixture. Additionally, the calculated ETC exhibited a decrease of $9.9 \%$ due to the effect of GF anisotropic thermal conductivity, and the ETC values decreased by $14.2 \%$ due to the effect of the interfacial thermal resistance at the Al-GF interface of the samples, which was determined to be in the range of 5.62$6.41 \times 10^{-8} \mathrm{~m}^{2} \mathrm{~K} \mathrm{~W}^{-1}$. [doi:10.2320/matertrans.MT-M2020071]
\end{abstract}

(Received February 26, 2020; Accepted October 27, 2020; Published December 25, 2020)

Keywords: graphite flakes/aluminum composites, orientation, interfacial thermal resistance, effective thermal conductivity, image-based simulation

\section{Introduction}

Electronic components are becoming smaller and have higher calculation speeds, owing to a rapid development of science and technology. Both high thermal conductivity (TC) and low coefficient of thermal expansion (CTE) are crucial properties in design of thermal-management materials (TMM). ${ }^{1-4)}$ Traditional TMMs, such as pure $\mathrm{Al}, \mathrm{Cu}, \mathrm{Au}$, and $\mathrm{Ag}$, do not comply with the development requirements of advanced electronic devices because they exhibit both high CTE and low TC.

Different carbon materials, such as diamond, carbon fiber $(\mathrm{CF})$, and graphite, have attracted considerable attention because of their high TC, low CTE, lightweight, and low cost. $^{3-6)}$ Several experiments have been performed to study the thermal properties of carbon-reinforced Al-matrix composites (diamond/Al, CFs/Al, and graphite flakes (GFs)/Al). However, these composites do not exhibit an ETC value as ultra-high as expected., ${ }^{4-15)}$

TC and CTE values for diamonds are 600-2300 $\mathrm{W} \mathrm{m}^{-1} \mathrm{~K}^{-14)}$ and $2.1 \times 10^{-6} \mathrm{~K}$, respectively. ${ }^{7)}$ The highest ETC of diamond/Al composites has been reported to be $670 \mathrm{~W} \mathrm{~m}^{-1} \mathrm{~K}^{-1}$. $)$ The reported ETC is significantly lower than the theoretical value because of the influence of the interfacial thermal resistance. Furthermore, weak wettability and interfacial bonding properties between diamond and the Al-matrix significantly reduce the ETC of the diamond/Al composite. These properties may be enhanced when diamonds undergo a surface treatment; however, such treatment is complicated, and diamonds are expensive materials. $^{7)} \mathrm{CF}$ exhibits TC values of 10 and 1100 $\mathrm{W} \mathrm{m}^{-1} \mathrm{~K}^{-1}$, along the radial direction ${ }^{5}$ and the axial direction, ${ }^{7)}$ respectively. $\mathrm{CF}$ is a one-dimensional material, and its TC has significant anisotropy. For CF-reinforced

\footnotetext{
${ }^{* 1}$ Graduate Student, Hiroshima University

${ }^{* 2}$ Corresponding author, E-mail: ksugio@hiroshima-u.ac.jp
}

Al-matrix composites, the high TC of CF does not have a significant contribution because of the non-controlled orientations of small $\mathrm{CFs}$ and interfacial reactions at the $\mathrm{CF}-\mathrm{Al}$ interface. GFs have a TC of $2200 \mathrm{~W} \mathrm{~m}^{-1} \mathrm{~K}^{-1}{ }^{8,9}$ ) and their CTE is $\sim 1.0 \times 10^{-6} \mathrm{~K}$ at the basal-plane. ${ }^{6)} \mathrm{GF} / \mathrm{Al}$ composites have been proposed as one of the most promising candidates as TMMs. Chen et al. ${ }^{13)}$ have reported a higher ETC for $80 \mathrm{vol} \% \mathrm{GF} / \mathrm{Al}$ composites $\left(783 \mathrm{~W} \mathrm{~m}^{-1} \mathrm{~K}^{-1}\right)$ compared to that for $80 \mathrm{vol} \%$ diamond $/ \mathrm{Cu}$ composites ${ }^{10)}$ $\left(724 \mathrm{~W} \mathrm{~m}^{-1} \mathrm{~K}^{-1}\right)$. Li et al. ${ }^{11)}$ obtained an ETC of 714 $\mathrm{W} \mathrm{m}{ }^{-1} \mathrm{~K}^{-1}$ for $70 \mathrm{vol} \% \mathrm{GF} / \mathrm{Al}$ composites in the plane parallel to the GF layers. However, it has been reported that GFs may exhibit strong anisotropic TC, i.e., $38 \mathrm{~W} \mathrm{~m}^{-1} \mathrm{~K}^{-1}$ along the out-of-plane direction. ${ }^{12)}$ The orientation of GFs significantly affects the ETC of the related composites. Although several studies have reported high ETC values for GF/Al composites, ${ }^{9,11,13)}$ a high TC similar to that of GFs $\left(2200 \mathrm{~W} \mathrm{~m}^{-1} \mathrm{~K}^{-1}\right)$ cannot be achieved for the composites because of the effect of the orientation of GFs.

In addition, the interfacial thermal resistance of the composites is considered to have a significant effect on their ETC. To predict the interfacial thermal resistance, Swartz and Pohl ${ }^{16)}$ proposed the diffuse mismatch model (DMM) for phonons. The DMM model assumes an ideal interface to calculate the interfacial thermal resistance. However, the actual interface differs from ideality and is complex. The interface is affected by crystal defects, as well as the inherent thermal properties of the starting materials, gaps at the interface, and fabrication methods. Therefore, the interfacial thermal resistance obtained from the DMM model is not sufficiently reliable.

To take advantage of the high TC of GFs and maximize the ETC of carbon-reinforced metal-matrix composites, it is crucial to investigate the effect of the anisotropic TC and the interfacial thermal resistance. In this study, GF/Al composites were prepared. To investigate the effect of the GF orientations and the interfacial thermal resistance on the ETC 
values for $\mathrm{GF} / \mathrm{Al}$ composites, a novel 2D image-based simulation method ${ }^{17)}$ was used to calculate the ETC of GF/ $\mathrm{Al}$ composites. In addition, accurate values of the interfacial thermal resistances at the Al-GF interface were obtained herein.

\section{Experimental and Calculation Procedures}

\subsection{Experiments}

Al powder $(99.9 \%, 30 \mu \mathrm{m}$ in diameter) and GFs $(98 \%$, average particle size: $137.02 \mu \mathrm{m}$ ) were used. The nominal TC of the Al powder was $236 \mathrm{~W} \mathrm{~m}^{-1} \mathrm{~K}^{-1}$, while that of GFs was 880 and $38 \mathrm{~W} \mathrm{~m}^{-1} \mathrm{~K}^{-1}$ at the basal-plane and out-ofplane direction, respectively. ${ }^{12)} \mathrm{Al}$ was mixed with $10 \mathrm{vol} \%$ and $20 \mathrm{vol} \%$ of GFs, respectively. Subsequently, appropriate amounts of alumina balls were added through a wet process for $2 \mathrm{~h}$ using a $\mathrm{V}$-type mixer at $50 \mathrm{rpm}$.

The resulting mixtures were filled into a graphite mold. The GFs stacked layer by layer into the mold after tapping. Then, spark plasma sintering (SPS) was conducted at $873 \mathrm{~K}$ and $60 \mathrm{MPa}$ pressure for $0.5 \mathrm{~h}$ under a vacuum of $1.3 \times$ $10^{-2} \mathrm{~Pa}$. To avoid the occurrence of chemical reaction at the Al-GF interface, the sintering temperature was maintained lower than the melting point of Al. The temperature was increased at a rate of $200 \mathrm{~K} / \mathrm{min}$. Al sample, $10 \mathrm{vol} \% \mathrm{GF} / \mathrm{Al}$ (Samples 1-2), and 20 vol\% GF/Al (Samples 3-4) samples were fabricated. The microstructures of the sintered samples were observed via optical microscopy. Figure 1 depicts the shape of the samples sintered via SPS and that of the microstructure images obtained in the $\mathrm{P} 1, \mathrm{P} 2$, and $\mathrm{P} 3$ regions at the A plane. The relative density was measured using the Archimedes method. ETC values were measured at $25^{\circ} \mathrm{C}$ using a steady-state thermal-conductivity analyzer.

\subsection{Numerical methods}

2D image-based simulations were performed in two steps. First, the ETCs of composites were recorded considering the effect of the orientations of GFs. Then, ETCs of the composites were calculated considering the orientations of the GFs and the interfacial thermal resistance at the interface between the $\mathrm{Al}$ matrix and GFs. The obtained microstructures of the experimental samples were used to perform 2D imagebased simulations.

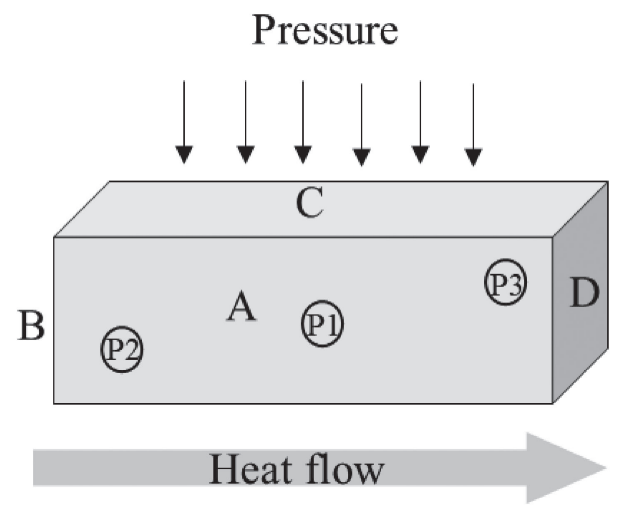

Fig. 1 Shape of the sample sintered by SPS; P1, P2, and P3 at the A plane denote the regions that correspond to the optical micrographs. The grey arrow illustrates that the heat flow was from B to D during ETC measurement, $\mathrm{C}$ is the plane bearing the pressure during sintering.

\subsubsection{Temperature distribution}

We calculated 2D-temperature distributions using the finite volume method as follows:

$T_{x, y}^{n+1}=T_{x, y}^{n}+\frac{\Delta t}{\rho c}\left(\frac{q_{x+1, y}^{n}-q_{x-1, y}^{n} q_{x-1, y}}{\Delta x}+\frac{q_{x, y+1}^{n}-q_{x, y-1}^{n}}{\Delta y}\right)$

where $x$ and $y$ denote the coordinate positions of the element, $T_{x y}^{n}$ is the temperature of the element at $(x, y)$ coordinates, and $T_{x, y}^{n+1}$ is the temperature of the element at $(x, y)$ coordinates after a time $\Delta t, \rho$ is the density, $\mathrm{c}$ is the specific heat, $\Delta x$ and $\Delta y$ denote the sizes of elements along the $x$-axis and $y$-axis, respectively, and $q^{n}$ denotes the heat flow. When $q^{n}$ is due to heat conduction between adjacent elements (e.g., as depicted in Fig. 2, $q^{n}$ moves from the $\mathrm{E}$ to adjacent elements $\mathrm{M}$ or $\mathrm{N}$ ), thus, $q^{n}$ can be calculated as follows:

$$
\begin{aligned}
& q_{x+1, y}^{n}=\lambda_{x+\frac{1}{2}, y}\left(\frac{T_{x+1, y}^{n}-T_{x, y}^{n}}{\Delta x}\right), \\
& q_{x-1, y}^{n}=\lambda_{x-\frac{1}{2}, y}\left(\frac{T_{x, y}^{n}-T_{x-1, y}^{n}}{\Delta x}\right) \\
& q_{x, y+1}^{n}=\lambda_{x, y+\frac{1}{2}}\left(\frac{T_{x, y+1}^{n}-T_{x, y}^{n}}{\Delta y}\right), \\
& q_{x, y-1}^{n}=\lambda_{x, y-\frac{1}{2}}\left(\frac{T_{x, y}^{n}-T_{x, y-1}^{n}}{\Delta y}\right) \\
& \lambda_{x+\frac{1}{2}, y}=\frac{2 \lambda_{x, y} \lambda_{x+1, y}}{\lambda_{x, y}+\lambda_{x+1, y}}, \quad \lambda_{x-\frac{1}{2}, y}=\frac{2 \lambda_{x, y} \lambda_{x-1, y}}{\lambda_{x, y}+\lambda_{x-1, y}} \\
& \lambda_{x, y+\frac{1}{2}}=\frac{2 \lambda_{x, y} \lambda_{x, y+1}}{\lambda_{x, y}+\lambda_{x, y+1}}, \quad \lambda_{x, y-\frac{1}{2}}=\frac{2 \lambda_{x, y} \lambda_{x, y-1}}{\lambda_{x, y}+\lambda_{x, y-1}}
\end{aligned}
$$

where $\lambda_{(x, y)}$ denotes the TC of the element at $(x, y)$ coordinates, $\lambda_{(x+1, y)}, \lambda_{(x-1, y)}, \lambda_{(x, y+1)}$, and $\lambda_{(x, y-1)}$ denote the TC of the elements adjacent to $(x, y)$, and $\lambda_{(x+1 / 2, y)}, \lambda_{(x-1 / 2, y)}$, $\lambda_{(x, y+1 / 2)}$, and $\lambda_{(x, y-1 / 2)}$ denote the harmonic mean of TC.

Considering the effect of the interfacial thermal resistance in the composite, if $q^{n}$ is due to the heat transfer between different types of elements, the interfacial heat transfer coefficient $(h)$ must be considered. As depicted in Fig. 2, considering that $q^{n}$ moves from $\mathrm{E}$ to $\mathrm{M}$ and through the

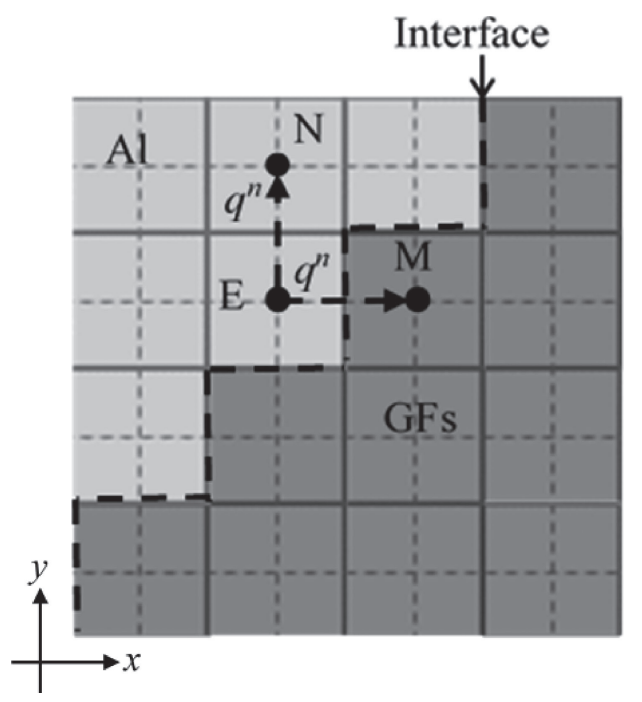

Fig. 2 Heat flow at the interface. The dashed line denotes the Al-GF interface, $q^{n}$ denotes the heat flow in the direction of the dashed arrows. 


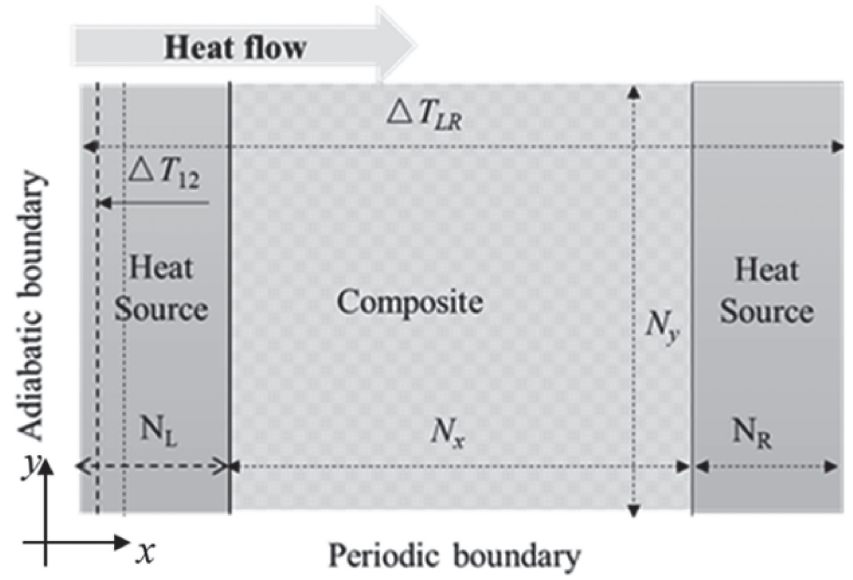

Fig. 3 Simulation model for the ETC calculation.

interface between $\mathrm{Al}$ and $\mathrm{GFs}(\mathrm{Al}-\mathrm{GF}), q^{n}$ can be calculated as follows:

$$
\begin{array}{ll}
q_{x+1, y}^{n}=h\left(T_{x+1, y}^{n}-T_{x, y}^{n}\right), & q_{x-1, y}^{n}=h\left(T_{x, y}^{n}-T_{x-1, y}^{n}\right) \\
q_{x, y+1}^{n}=h\left(T_{x, y+1}^{n}-T_{x, y}^{n}\right), & q_{x, y-1}^{n}=h\left(T_{x, y}^{n}-T_{x, y-1}^{n}\right)
\end{array}
$$

\subsubsection{Calculation of effective thermal conductivity}

As depicted in Fig. 3, the simulation model comprised two heat sources and composite components. Both the top and bottom surfaces correspond to the periodic boundary, while the left and right sides to the adiabatic boundary. The composite part is based on the microstructures of Samples $1-4$. Therefore, the size of the composite part $\left(N_{x} \times N_{y}\right)$ was $450 \times 600$ elements for Samples 1, 2, and 4, while it was $570 \times 450$ elements for Sample 3. The size of the heat sources was $5 \times 600$ elements for Samples 1, 2, and 4, and it was $5 \times 450$ elements for sample $3\left(N_{L}=N_{R}=5\right.$ elements). The size of each element was $1.18 \times 10^{-6} \mathrm{~m}$. The TCs of the GFs were set to $(880,38) \mathrm{W} \mathrm{m}^{-1} \mathrm{~K}^{-1}$ along the $(x, y)$ directions. However, the TC of the GFs was influenced by their orientation in the $\mathrm{Al}$ matrix. The TC calculation of GFs is presented in section 2.2.3, and the ETC calculation of the Al matrix is described in section 3.2.

The temperature of the left edge elements was set to $301 \mathrm{~K}$, and the initial temperature of the other elements was set to $300 \mathrm{~K}$. The temperatures at the left and right sides were set constant. The temperature of the remaining elements was iterated until the temperature variation was lower than $10^{-13} \mathrm{~K}$, and the temperature distribution in the steady state was obtained. Simulated ETC values of the GF/Al composites, $\lambda_{\text {s-eff, }}$ were calculated for the temperature distribution in the steady state as follows:

$$
\lambda_{\text {s-eff }}=\frac{\lambda_{A l-\text { eff }} \Delta T_{12} N_{x}}{\Delta T_{L R}-N_{L} \Delta T_{12}-N_{R} \Delta T_{12}}
$$

where $\lambda_{A l-e f f}$ denotes the TC of the Al matrix, and $\Delta T_{12}$ is the average temperature difference between the first and second columns; $N_{L}$ and $N_{R}$ denote the number of elements in the heat sources, $N_{x}$ and $N_{y}$ are the number of elements in the composite region.

\subsubsection{Anisotropic thermal conductivity of GFs}

It is well known that GFs exhibit a layered crystal structure. The atoms in the layer plane exhibit strong covalent bonds. However, the atoms between the layers are bonded

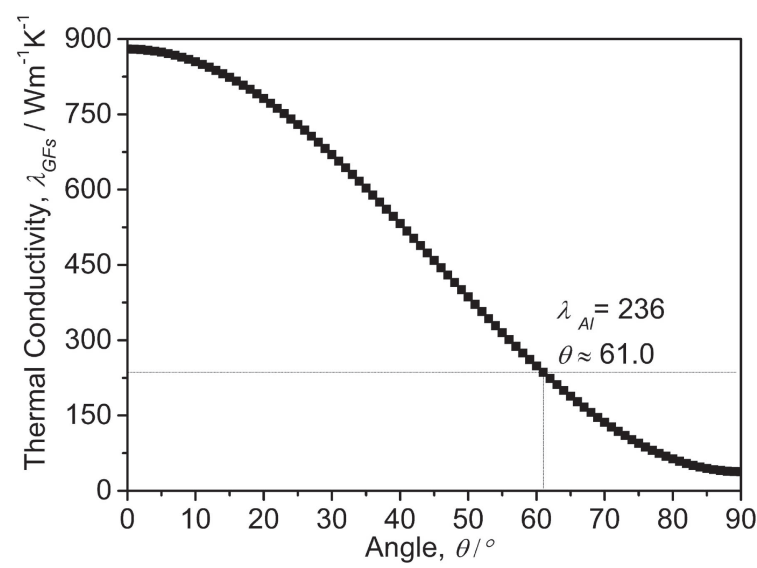

Fig. 4 TC of GFs as a function of the angle $(\theta)$. The nominal TC of Al matrix, $\lambda_{A l}$, was $236 \mathrm{~W} \mathrm{~m}^{-1} \mathrm{~K}^{-1}$ (gray horizontal dotted line).

via weak van der Waals forces. While, the thermal properties along the layer plane are excellent, they are low between the layers. Moreover, the TC of the GFs exhibits a strong anisotropy. In this study, the angle between the basal plane of the GFs and the heat flow direction denoted the orientation of the GFs in the Al matrix. The TCs in the direction parallel $\left(\lambda_{/ /}\right)$and perpendicular $\left(\lambda_{\perp}\right)$ to the heat flow direction can be calculated as follows: ${ }^{18)}$

$$
\begin{aligned}
& \lambda_{/ /}=\lambda_{a}\left[1-\left(1-\frac{\lambda_{c}}{\lambda_{a}}\right) \sin ^{2} \theta\right] \\
& \lambda_{\perp}=\lambda_{a}\left[1-\left(1-\frac{\lambda_{c}}{\lambda_{a}}\right) \cos ^{2} \theta\right]
\end{aligned}
$$

where $\lambda_{a}$ and $\lambda_{c}$ are the TC in the basal-plane and out-ofplane directions of the GFs, respectively. $\theta$ denotes the angle between the heat flow direction and basal-plane of the GFs.

Figure 4 illustrates the relationship between $\mathrm{TC}$ and the angle, $\theta$. It can be observed that TC decreased significantly from 880 to $38 \mathrm{~W} \mathrm{~m}^{-1} \mathrm{~K}^{-1}$ when the angle increased. The orientation of the GFs significantly affects the ETC of the composites. In addition, Fig. 4 shows that the TC of the GFs was lower than that of the $\mathrm{Al}$ matrix $\left(236 \mathrm{~W} \mathrm{~m}^{-1} \mathrm{~K}^{-1}\right)$ at the angles greater than $61^{\circ}$, which indicated that enhanced ETCs were obtained for critical angles smaller than or equal to $61^{\circ}$.

\section{Results and Discussion}

\subsection{Microstructures}

Figure 5 illustrates the microstructures of the samples. The GFs were homogeneously distributed in the Al matrix. However, GF orientations were not uniform in the samples. As shown in Fig. 5, the orientations of the GFs were almost parallel to each other in Samples 1 and 4. GF orientations were partial disordered in Samples 2 and 3, forming different angles between GFs basal-plane of the GFs and heat flow direction. The orientations of GFs in Fig. 5 were determined. As depicted in Fig. 6(a), the GFs were bounded by the smallest circumscribed rectangle, and the rectangles represent the orientation of the GFs. The angle $\theta$ with respect to the heat flow direction. The $\theta$ values in the microstructures were measured as depicted in Fig. 6(b), excluding GFs smaller than $5 \times 5$ pixels. The measured $|\theta|$ values are shown in Figs. 6(c)-(f). Most of the angles ranged from $0^{\circ}$ to $30^{\circ}$ in all 


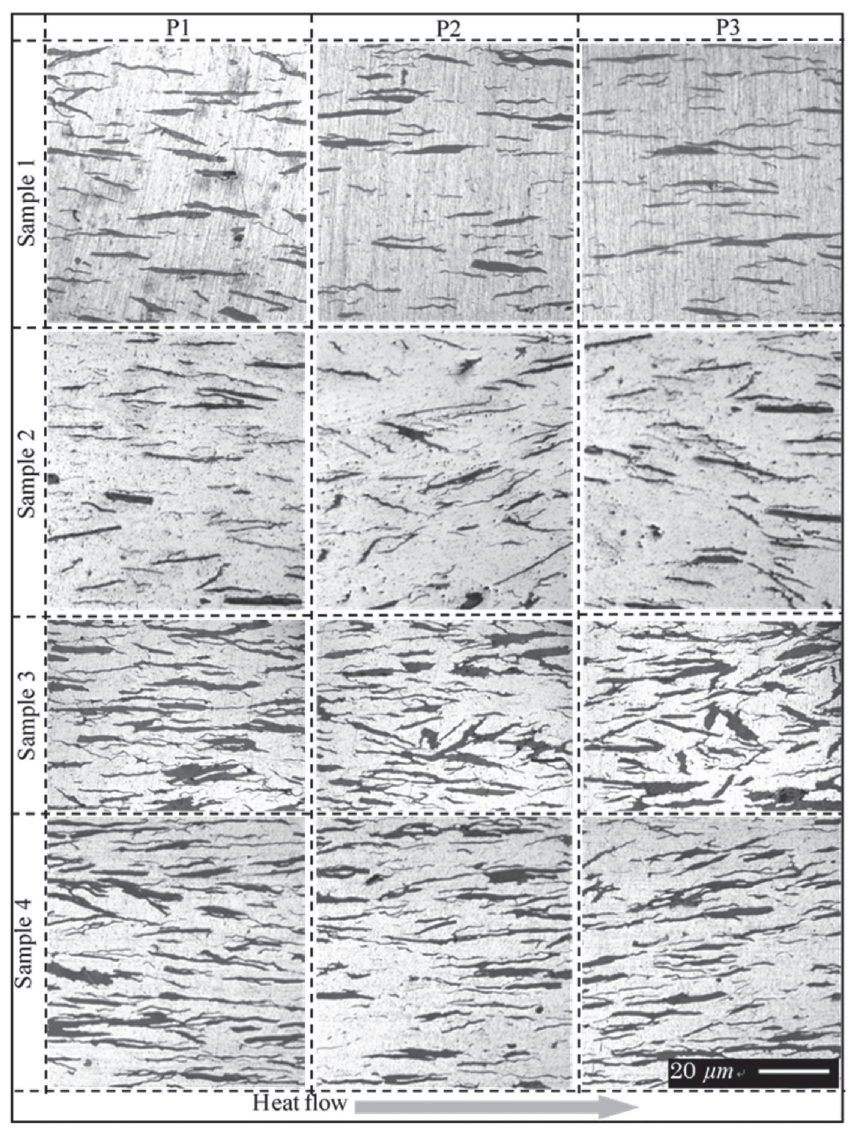

Fig. 5 Optical micrographs of Samples 1-4. Regions P1, P2, and P3 correspond to the black circles at the A-plane in Fig. 1. The gray arrow represents the heat flow direction during the ETC measurements. The brighter phases correspond to the Al matrix and the elongated dark phases to the GFs.

the samples. However, for the angles ranging from $50^{\circ}$ to $90^{\circ}$, the relative frequency in Samples 1 and 4 was $<10$, but it was $>10 \%$ in Samples 2 and 3. As depicted in Fig. 4, when the angle between the basal-plane of the GFs and the heat flow direction was $>61.0^{\circ}$, the TC of the GFs along the heat flow direction was smaller than that of the Al matrix. Thus, it can be derived from Fig. 6 that the average TCs of the GFs in Samples 2 and 3 along the heat flow direction were smaller than the average TCs of the GFs in Samples 1 and 4. The average angle for each sample was also calculated, as presented in Figs. 6(c)-(f).

\subsection{Measuring the ETC and relative density}

The measured ETCs and relative densities of the experimental samples are listed in Table 1. The ETC of Sample 1 was higher than that of Sample 2, and the ETC of Sample 3 was lower than that of Sample 4. These measurement results agree with the results shown in Fig. 6.

Relative density measurements (Table 1) indicate that some pores existed in the composites. In this study, the pores for all the samples were assumed to be in the $\mathrm{Al}$ matrix. To eliminate the pore effect on the TC, the following equation derived by Landauer ${ }^{19}$ ) was used:

$$
\begin{aligned}
\lambda_{A l-\text { eff }}= & \frac{1}{4}\left[\lambda_{p}\left(3 v_{p}-1\right)+\lambda_{A l}\left(3 v_{A l}-1\right)\right. \\
& \left.+\left(\left[\lambda_{p}\left(3 v_{p}-1\right)+\lambda_{A l}\left(3 v_{A l}-1\right)\right]^{2}+8 \lambda_{p} \lambda_{A l}\right)^{\frac{1}{2}}\right],
\end{aligned}
$$

where $\lambda_{A l-e f f}$ denotes the TC of the Al matrix, $\lambda_{A l}$ and $\lambda_{p}$ are the actual TCs of the material Al powder and pores, respectively, $v_{A l}$ is the volume fraction of $\mathrm{Al}$, and $v_{p}$ is the volume fraction of the pores. In addition, $v_{A l}, \mathrm{v}_{\mathrm{p}}$, and $\lambda_{\mathrm{p}}$ are listed in Table 2. First, the measured TC of the Al sample $\left(\lambda_{A l-e f f}, 217 \mathrm{~W} \mathrm{~m}^{-1} \mathrm{~K}^{-1}\right)$ and its relative density (97\%) were substituted in eq. (6), and $\lambda_{A l}$ was determined to be 228 $\mathrm{W} \mathrm{m}{ }^{-1} \mathrm{~K}^{-1}$, as shown in Table 2. Second, the TC of the Almatrix for Samples $1-4, \lambda_{\text {Al-eff, }}$ was calculated by substituting $v_{A l}, v_{p}, \lambda_{p}$, and $\lambda_{A l}$ from Table 2 in eq. (6). The calculated values of $\lambda_{\text {Al-eff }}$ are $225.3,225.9,221.2$, and $225.6 \mathrm{~W} \mathrm{~m}^{-1} \mathrm{~K}^{-1}$ for Samples 1-4, respectively. The ETCs $\left(\lambda_{R O M}\right)$ of Samples 1-4 were calculated using the rule of mixture $(R O M)$, i.e., $\lambda_{R O M}=\left(v_{G F S}\right)\left(\lambda_{G F S}\right)+\left(1-v_{G F s}\right)\left(\lambda_{A l-e f f}\right)$, where the orientations of all the GFs were assumed to be aligned with the heat flow direction in the composites. The TC of the GFs $\left(\lambda_{G F s}\right)$ was set to $880 \mathrm{~W} \mathrm{~m}^{-1} \mathrm{~K}^{-1}$ along the heat flow direction. The values of $\lambda_{R O M}$ were $291,291,353$, and $357 \mathrm{~W} \mathrm{~m}^{-1} \mathrm{~K}^{-1}$ for Samples $1-4$, respectively. The calculated $\lambda_{R O M}$ was considerably higher than the measured ETC. Furthermore, to confirm the effect of the orientation of the GFs on ETC for the composites, we performed 2D image-based simulations.

\subsection{Effect of the orientation of GFs on ETC}

The TC values of GFs in the experimental samples were calculated using eq. (5); Fig. 7 illustrates the results. GFs are denoted in different colors according to the orientations of the GFs in the Al matrix. When the orientation of the GFs was parallel to the heat flow direction, the GFs exhibited high TC and, accordingly, they were marked in red. However, if the GFs had a tilt from the heat flow direction, color changes indicated a decrease in TC. Figure 7 shows that most GFs were colored in red for Samples 1 and 4, which implied that GFs had a high TC, while the yellow, blue, and other colors appeared in the P2 and P3 regions for Samples 2 and 3.

We performed a 2D image-based simulation, and the simulated ETCs $\left(\lambda_{s-e f f}\right)$ are listed in Table 2 . Figure 8 shows the measured ETC, $\lambda_{R O M}$, and $\lambda_{s-e f f}$ of Samples $1-4$. The $\lambda_{s-e f f}$ values were smaller than those of $\lambda_{R O M}$, and the deviations between $\lambda_{R O M}$ and $\lambda_{\text {s-eff }}$ were $7.2 \%, 9.6 \%, 9.9 \%$, and $9.5 \%$ for Samples 1-4, respectively. The difference in the ETC loss between Samples 1 and 2 was $2.4 \%$, which is greater than the ETC loss of $0.4 \%$ between Samples 3 and 4 . This can be attributed to the deviations in the average angle between the GF basal-plane and the heat flow direction, as depicted in Figs. 6(c)-(f). Moreover, the deviation between Samples 1 and $2\left(17^{\circ}\right)$ was larger than that between Samples 3 and 4 $\left(3^{\circ}\right)$.

As shown in Fig. 8, $\lambda_{s-\text { eff }}$ values were higher than measured ETC values, and that the deviations between $\lambda_{\text {s-eff }}$ and the measured ETCs were $8.1 \%, 9.5 \%, 14.2 \%$, and $13.3 \%$ for Samples $1-4$, respectively. These deviations can be attributed to the effect of the interfacial thermal resistance between $\mathrm{Al}$ and GFs.

\subsection{Effect of the interfacial thermal resistance on the ETC}

A series of 2D image-based simulations were performed to study the effect of the interfacial thermal resistance on the ETC of the composites. The heat transfer coefficient, $h$, 

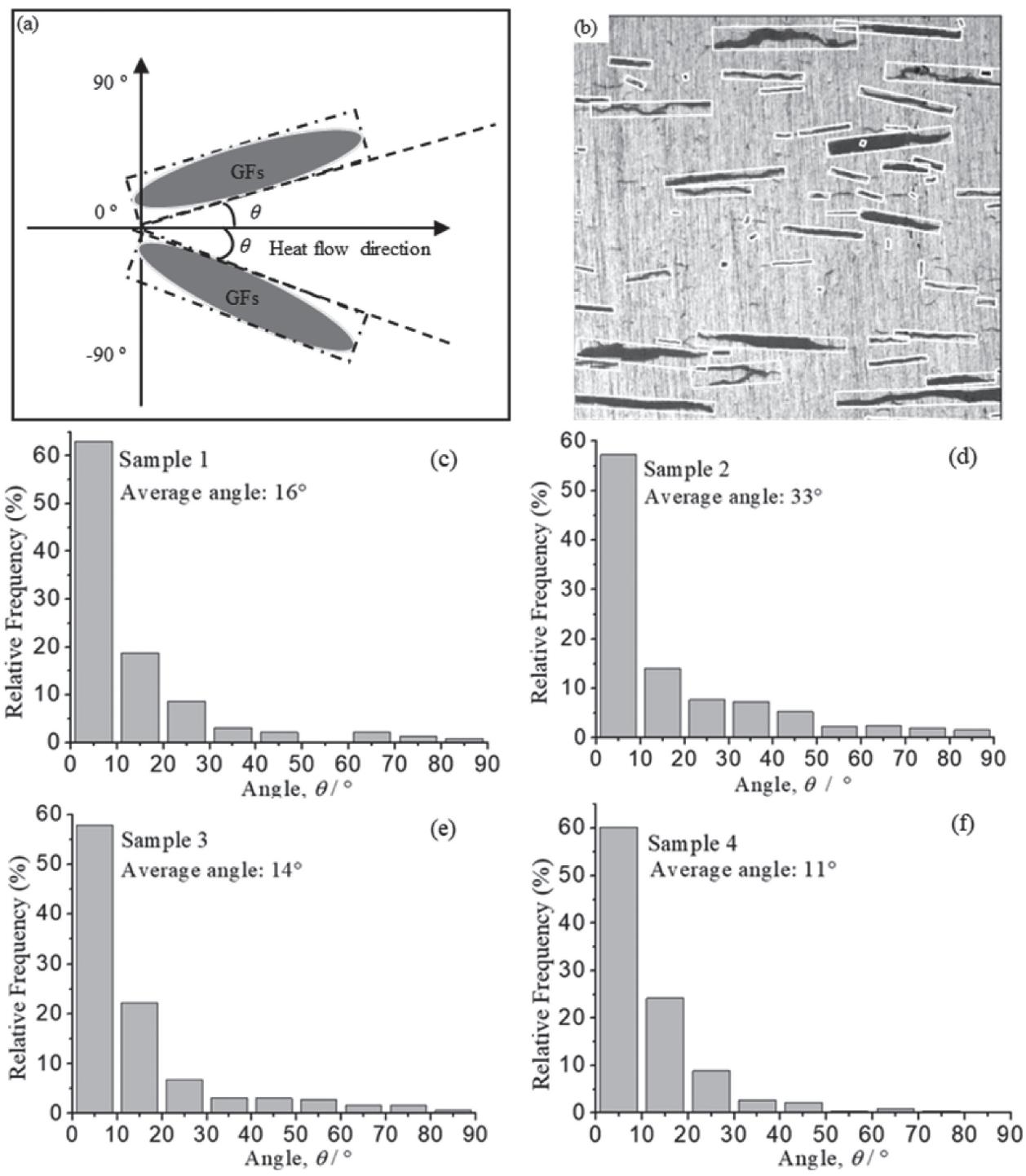

Fig. 6 Distribution of the GF orientations in the Al matrix. Gray and white rectangles in (a) and (b) represent the smallest circumscribed rectangles and were used to measure the angle $(\theta)$ between the basal-plane of GFs and the heat flow direction; in case $-90<\theta<0$, $\theta$ was taken as the absolute value.

Table 1 Volume fractions of GFs $\left(v_{G F s}\right)$, measured ETCs $\left(\lambda_{e f f}\right)$, and relative densities of Samples 1-4 and pure Al sample.

\begin{tabular}{cccc}
\hline \multirow{2}{*}{ Sample } & $v_{G F s}$ & $\lambda_{\text {eff }}$ & \multirow{2}{*}{ Relative density } \\
\cline { 2 - 4 } & $\%$ & $\mathrm{Wm}^{-1} \mathrm{~K}^{-1}$ & $99.2 \%$ \\
1 & 10 & 248 & $99.4 \%$ \\
2 & 10 & 238 & $98.0 \%$ \\
3 & 20 & 273 & $99.3 \%$ \\
4 & 20 & 280 & $97.0 \%$ \\
$\mathrm{Al}$ & 0 & 217 & \\
\hline
\end{tabular}

ranged from $10^{3}$ to $10^{9} \mathrm{~W} \mathrm{~m}^{-2} \mathrm{~K}^{-1}$ at the $\mathrm{Al}-\mathrm{GF}$ interface. Figure 9 illustrates ETC values as a function of $h$ for composites. The ETC curve was similar with $\mathrm{KJMA}^{20)}$ equation and ETC values increased rapidly as $h$ increased from $10^{5}$ to $10^{9}$. Moreover, ETC curves for Sample 1 and Sample 2 did not overlap in the $h$ values range from $10^{3}$ to $10^{9}$, further proving that the orientations of the GFs significantly influenced the ETC values of the composites. In Fig. 9, the dashed lines represent the experimental ETC and the arrows denote the heat transfer coefficients at the Al-GF interface.
Table 2 Volume fractions of $\mathrm{Al}$ and the pores in the matrix $\left(v_{A l}, v_{p}\right)$, TC of air $\left(\lambda_{p}\right)$, actual TC of the Al sample $\left(\lambda_{A l}\right)$ without the effect of pores, TCs of the Al matrixes and the Al sample including the pore effect $\left(\lambda_{A l-e f f}\right)$, ETCs of Samples 1-4 calculated using ROM $\left(\lambda_{R O M}\right)$, and ETCs of Samples 1-4 $\left(\lambda_{s-e f f}\right)$ calculated using 2D simulations considering the anisotropic TC of the GFs.

\begin{tabular}{|c|c|c|c|c|c|c|c|}
\hline \multirow{2}{*}{ Sample } & \multirow{2}{*}{$v_{A l}$} & \multirow[b]{2}{*}{$v_{p}$} & $\lambda_{p}$ & $\lambda_{A l}$ & $\lambda_{\text {Al-eff }}$ & $\lambda_{R O M}$ & $\lambda_{\text {seff }}$ \\
\hline & & & \multicolumn{5}{|c|}{$\mathrm{Wm}^{-1} \mathrm{~K}^{-1}$} \\
\hline $\mathrm{Al}$ & 0.97 & 0.03 & 0.214 & 228 & 217 & - & - \\
\hline 1 & 0.992 & 0.008 & - & - & 225.3 & 291 & 270 \\
\hline 2 & 0.994 & 0.006 & 0.214 & - & 225.9 & 291 & 263 \\
\hline 3 & 0.98 & 0.02 & - & - & 221.2 & 353 & 318 \\
\hline 4 & 0.993 & 0.007 & - & - & 225.6 & 357 & 323 \\
\hline
\end{tabular}

Table 3 shows $h$ values evaluated via inverse analysis, which were in the same order of magnitude for all samples, i.e., $10^{7}$. An $h$ reference value was calculated using the DMM model, as listed in Table 3. The order of magnitude of Samples 1-4 was in agreement with that of the reference value. The observed small differences between the $h$ 


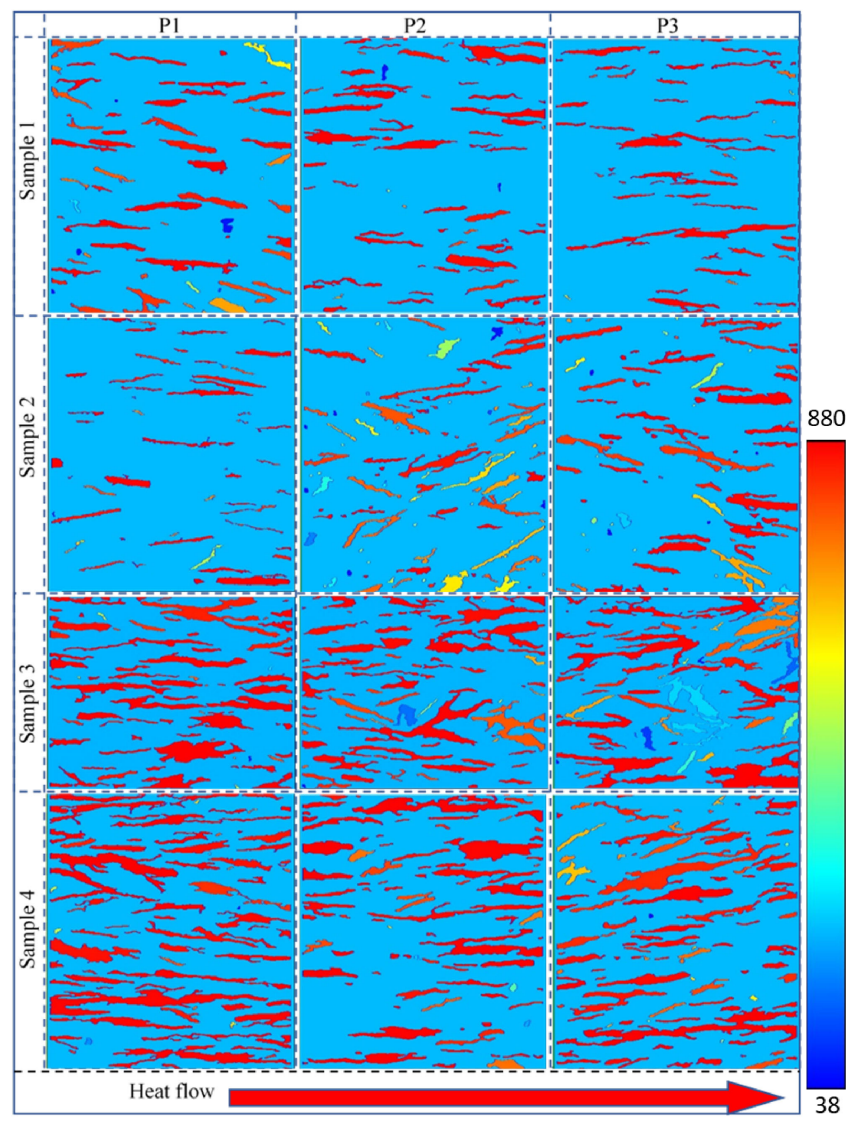

Fig. 7 TC values of GFs in Samples1-4. Regions denoted as P1, P2, and P3 correspond to the microstructures in Fig. 5; the color bar shows the TCs of GFs in different orientations, where blue at the bottom of the color bar represents a $\mathrm{TC}$ of $38 \mathrm{~W} \mathrm{~m}^{-1} \mathrm{~K}^{-1}$, and red at the top of the color bar represents a TC of $880 \mathrm{~W} \mathrm{~m}^{-1} \mathrm{~K}^{-1}$. The red arrow indicates the heat flow direction.

Table 3 Heat transfer coefficient, $h$, at the interface between $\mathrm{Al}$ matrix and GFs ( $R$ denotes the interfacial thermal resistance).

\begin{tabular}{cccc}
\hline \multirow{2}{*}{ Sample } & \multicolumn{2}{c}{$h / \mathrm{W} \mathrm{m}^{-2} \mathrm{~K}^{-1}$} & \multirow{2}{*}{$R / \mathrm{m}^{2} \mathrm{~K} \mathrm{~W}^{-1}$} \\
\cline { 2 - 3 } & \multicolumn{2}{c}{ Cal. } & Ref. \\
2 & $1.62 \times 10^{7}$ & $\left.4.8 \times 10^{7} \mathrm{a}\right)$ & $6.17 \times 10^{-8}$ \\
2 & $1.56 \times 10^{7}$ & & $6.41 \times 10^{-8}$ \\
4 & $1.64 \times 10^{7}$ & & $6.10 \times 10^{-8}$ \\
& $1.78 \times 10^{7}$ & & $5.62 \times 10^{-8}$ \\
\hline
\end{tabular}

a) Reported value in reference (21)

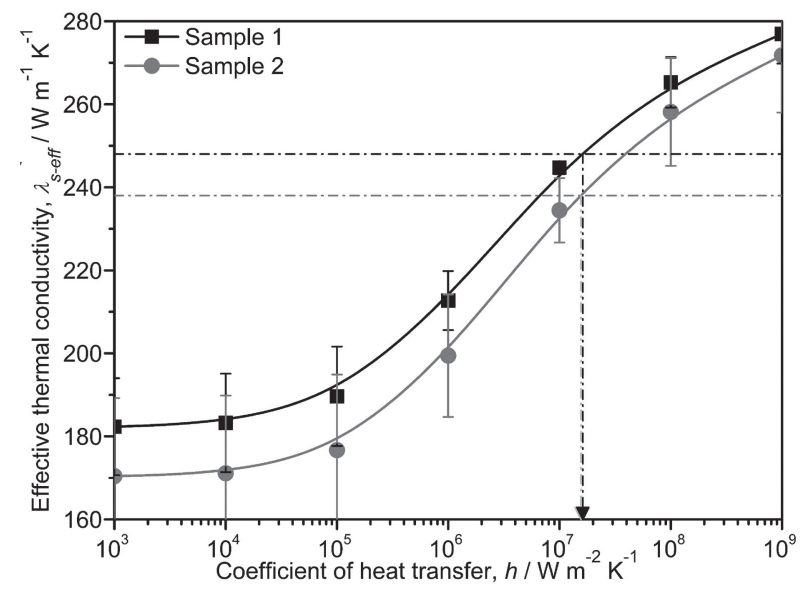

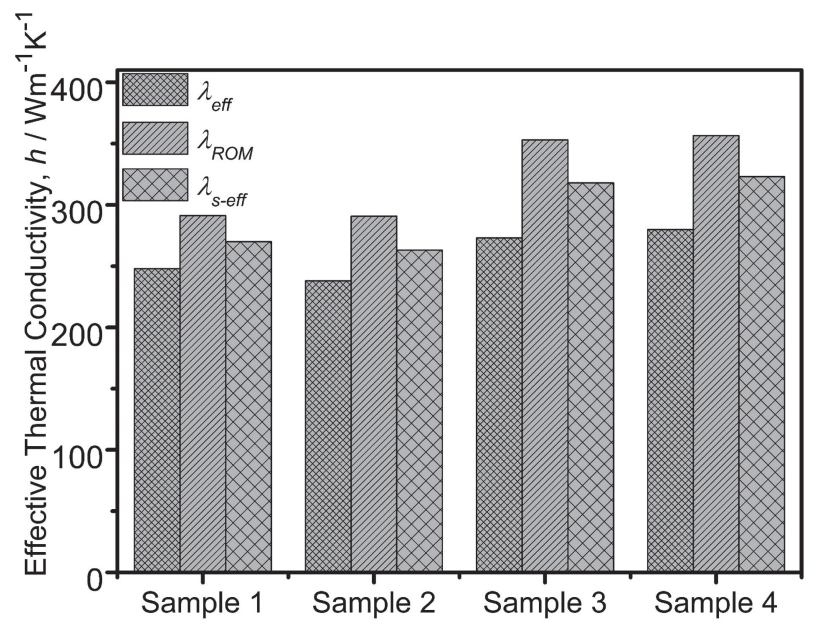

Fig. 8 ETCs of the composite samples. $\lambda_{\text {eff }}$ denotes the measured ETC, $\lambda_{R O M}$ is the ETC calculated using the rule of mixture, and $\lambda_{s-e f f}$ is the ETC calculated using the 2D image-based simulation considering GF orientations.

reference value and simulated $h$ for Samples $1-4$ were attributed to the misfits at the interface.

The interfacial thermal resistance, $R$, is the reciprocal of the heat transfer coefficient, i.e., $R=1 / h, R$ values for Samples 1-4 are listed in Table 3. It should be noted that $R$ values were sufficiently small and even negligible. However, as shown in Fig. 8, $R$ significantly affected the ETC of the composites, leading to a significant decrease in ETC. This can be attributed to the considerable number of interfaces formed in the GF/Al composites.

\section{Conclusions}

$10 \mathrm{vol} \% \mathrm{GF} / \mathrm{Al}$ and $20 \mathrm{vol} \% \mathrm{GF} / \mathrm{Al}$ composites were fabricated via SPS. The microstructures of the composites were observed and their relative density and ETCs were measured. The measured ETC values were 248, 238, 273, and $280 \mathrm{~W} \mathrm{~m}^{-1} \mathrm{~K}^{-1}$ for Samples $1-4$, which were smaller than the ETCs calculated through the $\operatorname{ROM}(291,291,353$, and $357 \mathrm{~W} \mathrm{~m}^{-1} \mathrm{~K}^{-1}$, for Samples $1-4$, respectively).

The average angles of the GFs with respect to the heat flow direction were calculated to be $16^{\circ}, 33^{\circ}, 14^{\circ}$, and $11^{\circ}$ for

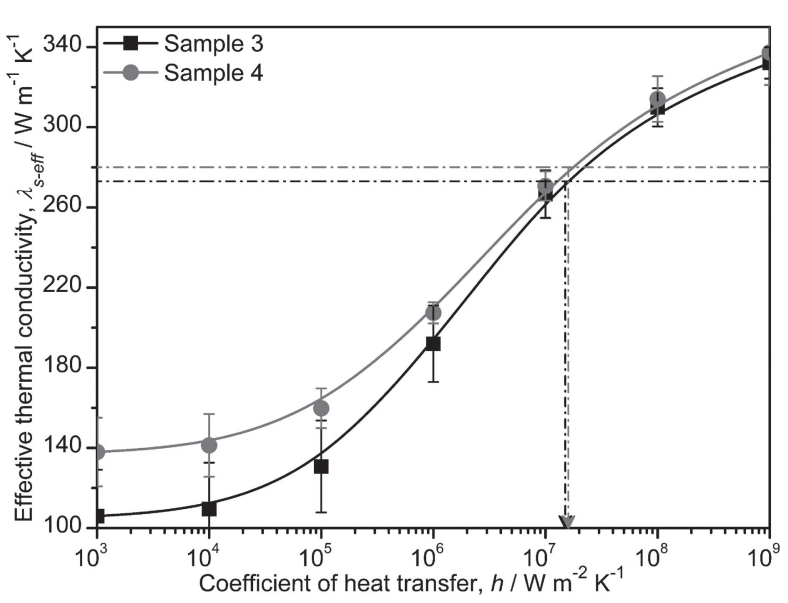

Fig. 9 ETC as a function of the heat transfer coefficient, $h$. The dashed lines represent the experimental ETCs and the arrows denote the $h$ value at the Al-GF interface. 
Samples 1-4, respectively. The ETCs of composites considering the orientations of GFs were evaluated via 2D imagebased simulation. Compared with the ETC calculated using $R O M$, the results showed that ETCs decreased due to the anisotropic TC of the GFs by $7.2 \%, 9.6 \%, 9.9 \%$, and $9.5 \%$ for Samples 1-4, respectively. The effect of the interfacial thermal resistance on ETCs was derived by comparing it with measured ETC values; it was observed that ETCs decreased by $8.1,9.5,14.2$, and $13.3 \%$ for Samples $1-4$, respectively. The $R$ values at the Al-GF interface was evaluated to be $6.17 \times 10^{-8}, 6.41 \times 10^{-8}, 6.10 \times 10^{-8}$, and $5.62 \times 10^{-8}$ $\mathrm{W} \mathrm{m}^{-2} \mathrm{~K}^{-1}$ for Samples $1-4$, respectively.

\section{REFERENCES}

1) Y. Choi, Y. Kataoka, Z. Zu, K. Matsugi and G. Sasaki: Mater. Trans. 58 (2017) 1097-1099.

2) M. Lee, Y. Choi, K. Sugio, K. Matsugi and G. Sasaki: Mater. Trans. 52 (2011) 939-942.

3) Y. Huang, Q. Ouyang, Q. Guo, X. Guo, G. Zhang and D. Zhang: Mater. Des. 90 (2016) 508-515.

4) X.-h. Qu, L. Zhang, M. Wu and S.-b. Ren: Prog. Nat. Sci.: Mater. Int 21 (2011) 189-197.

5) T. Tokunaga, K. Takahashi, M. Ohno, K. Sasaki, T. Imanishi and K.
Matsuura: Mater. Trans. 58 (2017) 938-944.

6) R. Prieto, J. Molina, J. Narciso and E. Louis: Scr. Mater. 59 (2008) 1114.

7) H. Kurita, T. Miyazaki, A. Kawasaki, Y. Lu and J.-F. Silvain: Compos., Part A 73 (2015) 125-131.

8) J. Norley: Electronics Cooling 7 (2001) 50-51.

9) S. Zhou, S. Chiang, J. Xu, H. Du, B. Li, C. Xu and F. Kang: Carbon 50 (2012) 5052-5061.

10) K. Yoshida and H. Morigami: Microelectron. Reliab. 44 (2004) 303308.

11) W. Li, Y. Liu and G. Wu: Carbon 95 (2015) 545-551.

12) R. Prieto, J.M. Molina, J. Narciso and E. Louis: Compos., Part A 42 (2011) 1970-1977.

13) J. Chen and I. Huang: Compos., Part B 44 (2013) 698-703.

14) G. Yuan, X. Li, Z. Dong, A. Westwood, Z. Cui, Y. Cong, H. Du and F. Kang: Carbon 50 (2012) 175-182.

15) C. Zhou, W. Huang, Z. Chen, G. Ji, M.L. Wang, D. Chen and H.W. Wang: Compos., Part B 70 (2015) 256-262.

16) E.T. Swartz and R.O. Pohl: Rev. Mod. Phys. 61 (1989) 605-668.

17) K. Sugio, Y.-B. Choi and G. Sasaki: Mater. Trans. 57 (2016) 582-589.

18) B.T. Kelly: Physics of Graphite, (Applied Science Publishers, London, 1981).

19) R. Landauer: J. Appl. Phys. 23 (1952) 779-784.

20) K. Sugio, S. Tatsuno, H. Fukushima and O. Yanagisawa: Mater. Trans. 50 (2009) 1563-1571.

21) C. Xue, H. Bai, P.F. Tao, J.W. Wang, N. Jiang and S.L. Wang: Mater. Des. 108 (2016) 250-258. 